\title{
The fms21 (pilA)-fms20 locus encoding one of four distinct pili of Enterococcus faecium is harboured on a large transferable plasmid associated with gut colonization and virulence
}

In the last two decades, enterococci, especially Enterococcus faecalis and Enterococcus faecium, have emerged as multidrug-resistant opportunistic pathogens causing difficult-to-treat healthcare-associated (HA) infections including endocarditis (Arias \& Murray, 2009). Recent surveillances reported a major epidemiological shift of increasing frequency of isolation of E. faecium from United States hospitals (Hidron et al., 2008). Furthermore, increased morbidity and mortality due to vancomycin-resistant strains of E. faecium have also been reported in patients who have multiple medical problems, such as cancer, transplantation or prior surgery. Using multilocus sequence typing, epidemiological studies have shown that the HA E. faecium strains are different sequence types from the commensal strains that colonize the gastrointestinal tract of healthy humans (Top et al., 2008). Further analyses suggested that the recent success of the E. faecium hospital-associated genogroup [also referred to as clonal complex 17 (CC17); Top et al., 2008] is due at least in part to the presence of an active form of the acm gene encoding collagen adhesin (Nallapareddy et al., 2008) or acquisition of additional genetic loci encoding enterococcal surface protein (esp $p_{f m}$ ) (Willems et al., 2001), a hyaluronidase-like glycoside hydrolase $\left(h y l_{E f m}\right.$ ) (Rice et al., 2003) or E. faecium surface proteins $(\mathrm{fms})$ (Hendrickx et al., 2007; Sillanpaa et al., 2008, 2009).

Pili and MSCRAMMs (microbial surface components recognizing adhesive matrix molecules) have been implicated in the ability of pathogenic bacteria to adhere to and colonize host tissues, processes important in initiating infections. Recent bioinformatics analyses of the unfinished genome sequence of endocarditis-derived E. faecium strain TX0016 (DO) identified 15 of 22 Fms proteins containing
MSCRAMM-like characteristics as well as predicted folding into multiple immunoglobulin-like domains (Sillanpaa et al., 2008). Of these, $11 \mathrm{fms}$ genes were found clustered in four genomic loci: $f m s 1$ $\left(e b p A_{f m}\right)-f m s 5\left(e b p B_{f m}\right)-f m s 9\left(e b p C_{f m}\right)$, fms11-fms19-fms16, fms14-fms17-fms13 and fms21 (pilA)-fms20, and each of these four loci also encodes a class $\mathrm{C}$ (subfamily 3) sortase predicted to be involved in pilus biogenesis (Sillanpaa et al., 2008). The proteins encoded by the $e b p_{f m}$ genes are orthologues of E. faecalis Ebp pili subunit proteins associated with pathogenesis in experimental rat endocarditis and mouse urinary tract infections (Nallapareddy et al., 2006; Singh et al., 2007). The fms11fms19-fms16 genes (designated the orf903907 gene cluster in Hendrickx et al., 2007) are present on a genomic island flanked by direct repeats, are often found as pseudogenes, and are enriched in the CC17 genogroup (Hendrickx et al., 2007; Sillanpaa et al., 2009). EbpC $\mathrm{fm}_{\mathrm{fm}}$ (designated PilB in Hendrickx et al., 2008) and Fms21 (designated PilA in Hendrickx et al., 2008) have been shown experimentally to form two distinct types of E. faecium pili (Hendrickx et al., 2008; Sillanpaa et al., 2008). However, the function(s) of these pili are yet to be elucidated.

In our current efforts of sequencing and analysis of the regions surrounding these pilus-encoding loci of TX0016 and TX1330 (a commensal human isolate), we identified a repA gene in the vicinity of the pilA (fms21)-fms20 locus, suggesting that this locus is likely harboured on a plasmid. The genes encoding PilA (Fms21) and Fms20 of these two strains were separated by two ORFs: SrtC4 and a 252 aa ORF with no cell-wall-anchoring motif exhibiting $>25 \%$ similarity to the $E$. faecalis EbpB pilus subunit protein. One class A sortase-encoding gene was also found immediately upstream of pilA ( $f m s 21)$ in both these strains. To provide experimental proof for the predicted plasmid localization of the pilA (fms21)fms20 locus, we performed Aspergillus oryzae S1 nuclease (Sigma) digests of agarose plugs containing genomic DNA of TX0016, TX1330 and TX2158 (a derivative of TX1330RF to which the $h y l_{E f m^{-}}$ containing plasmid was transferred by conjugation from TX0016; Arias et al., 2009) and four additional endocarditisderived E. faecium strains using a method described earlier (Arias et al., 2009). The S1 nuclease digestion combined with PFGE was previously shown to be useful for detection and estimation of the size of large bacterial plasmids (Barton et al., 1995). The isolates used in this study were selected based on pilA ( $f m s 21$ ), fms20 and $h y l_{E f m}$ (a marker for a transferable plasmid of TX0016; Arias et al., 2009) gene diversity that we previously reported using colony hybridization (Rice et al., 2003; Sillanpaa et al., 2008, 2009); the genotypes of these strains are shown in Table 1.

PFGE followed by Southern hybridizations showed that pilA ( $f m s 21)$ and $f m s 20$ are indeed located on plasmids of varying sizes, running between 145.5 and $291 \mathrm{~kb}$ in PFGE gels, in different E. faecium strains (Fig. 1). Southern hybridization profiles for these isolates are in agreement with our previously published colony hybridization data (Sillanpaa et al., 2008, 2009). The extrachromosomal location of pilA ( $f m s 21$ ) and $f m s 20$ genes was further confirmed by I-CeuI (which recognizes a sequence in $23 \mathrm{~S}$ rRNA genes, thus hybridization with a probe targeting 23S rRNA genes results in six chromosomal bands in each strain) digestion of agarose plugs followed by PFGE and hybridization (data not shown). In a separate experiment, EcoRV endonuclease digestion of caesium chloride-extracted, $h y l_{E f m}$-containing plasmid ( 220 kb) DNA from E. faecium strain TC6 (Rice et al., 2009) was performed. Southern hybridizations with 
Table 1. Characteristics of E. faecium isolates used in this study

\begin{tabular}{|c|c|c|c|c|c|}
\hline Strain & Clinical source; origin; year of isolation/collection & MLST type & $h y l_{E f m}{ }^{*}$ & pilA $(f m s 21)^{*}$ & $f m s 20^{*}$ \\
\hline TX0016† & Endocarditis/blood; Houston, TX, USA; 1992 & ST18 & + & + & + \\
\hline TX1330RF & $\begin{array}{l}\text { Rifampicin and fusidic acid resistant version of commensal } \\
\text { human isolate TX1330 }\end{array}$ & & - & + & + \\
\hline TX2158 & Transconjugant of TX1330RF-TX0016 & & + & + & + \\
\hline TX0068 & Endocarditis/blood; Worcester, MA, USA; 1994 & ST18 & - & + & + \\
\hline TX0074 & Endocarditis/blood; Valhalla, NY, USA; 1995 & ST337 & - & + & - \\
\hline TX0081 & Endocarditis/blood; Baltimore, MD, USA; 1996 & ST154 & + & + & - \\
\hline TX0082 & Endocarditis/blood; Houston, TX, USA; 1999 & ST17 & - & - & - \\
\hline
\end{tabular}

${ }^{*}+$, Gene present; -, gene absent.

$\dagger$ Also designated DO in previous studies.

$¥$ These isolates were generated in an earlier study (Arias et al., 2009).

pilA (fms21) and fms20 probes of these restriction-digested fragments showed that the $h y l_{E f m}$ and $f m s$ genes are co-localized in this plasmid as well (data not shown). We have also investigated the location of another CC17 genogroup-enriched (Hendrickx et al., 2007; Sillanpaa et al., 2009) gene cluster, fms11-fms19-fms16, by hybridization of Southern blots of $\mathrm{S} 1$ nuclease-digested DNA as well as I-CeuIdigested DNA with $f m s 11$, fms 16 and $f m s 19$ gene probes. The fms 11, fms 16 and $f m s 19$ genes were found to be on the chromosome of four of four isolates tested and were not present in the transconjugant strain TX2158, indicating that they were not acquired along with transfer of the $h y l_{E f m}$ plasmid.
Of note, we also analysed the pilA ( $f m s 21)$ fms20 surrounding regions of multiple unfinished E. faecium whole genome sequences (recently sequenced by the Broad Institute of MIT and Harvard, Cambridge, MA, USA, and available at http://www.ncbi.nlm.nih.gov/sutils/ genom_table.cgi) belonging to clinical and non-clinical groups and found repA in the
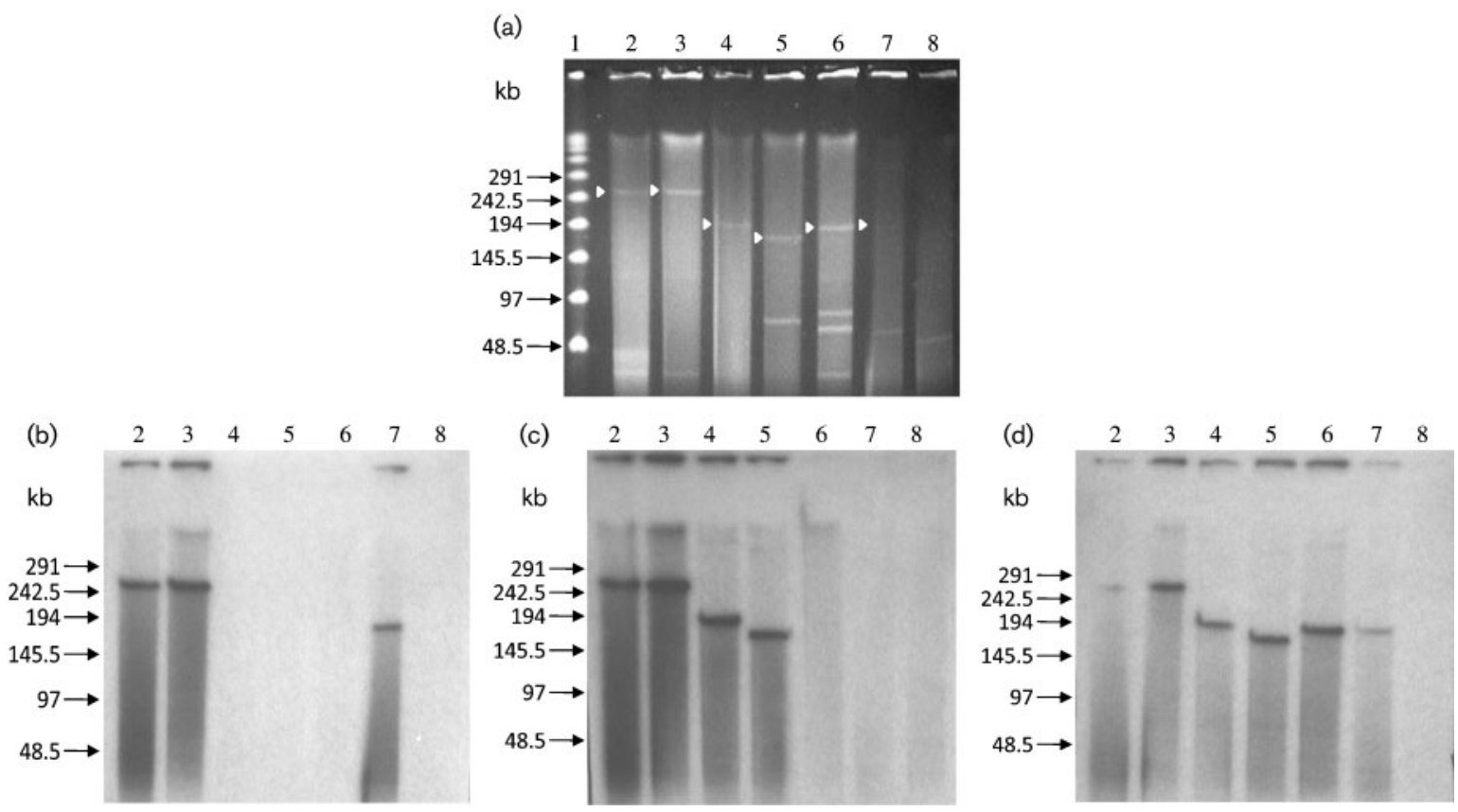

Fig. 1. PFGE of $S 1$ nuclease digested genomic DNA showing large plasmids of $E$. faecium isolates and hybridizations with $h y l_{E f m}$, pilA (fms21) and fms20 probes. (a) PFGE of S1 digestions of total genomic DNA. Plasmid bands are shown as linearized fragments on the gel; the white arrows represent the plasmid bands hybridizing with the different probes tested in the following panels. (b) Hybridizations with the hyl $l_{E f m}$ probe. (c) Hybridizations with the fms20 probe. (d) Hybridizations with the pilA (fms21) probe. Lanes: 1, lambda ladder; 2, TX0016; 3, TX2158; 4, TX1330RF; 5, TX0068; 6, TX0074; 7, TX0081; 8, TX0082. 
neighbourhood [present in the same contig as pilA (fms21)-fms20], further confirming that the pilA locus is harboured on a plasmid in multiple genetic backgrounds.

In E. faecium strains that are $h y l_{E f m}{ }^{+}$ (TX0016, TX2158 and TX0081), pilA (fms21) and/or fms20 probes as well as an $h y l_{E f m}$ probe hybridized to same plasmid band ( 250 kb plasmid in TX0016 as well as in the TX2158 transconjugant and to $\sim 170 \mathrm{~kb}$ plasmid in TX0081).

Interestingly, as shown in Fig. 1, transconjugant TX2158 lost the TX1330RF native plasmid (of size $\sim 190 \mathrm{~kb}$ ) during acquisition of the $h y l_{E f m}$ plasmid from donor TX0016, possibly due to incompatibility. Sequence analysis of an $\sim 45 \mathrm{~kb}$ region spanning pilA (fms21)fms 20 and the repA region of the native plasmids of TX1330 and TX0016 showed $>90 \%$ identity (unpublished results).

Although $h y l_{E f m}$ has been implicated as a possible virulence determinant in multiple worldwide surveys based on its enrichment in the HA genogroup, the exact role of the $h y l_{E f m}$ gene cluster (flanked by insertion elements) in pathogenesis or colonization by E. faecium has not been demonstrated. However, it has been recently reported that it is encoded on a large transferable plasmid containing variable antibiotic resistance genes in different isolates (Arias et al., 2009) and the presence of this plasmid enhances the ability of E. faecium to colonize the gut of mice (Rice et al., 2009) and increases lethality in a mouse peritonitis model (Arias et al., 2009).

Taken together, we speculate that some ancestral and commensal isolates carried the pilA (fms21)-fms20 gene cluster on a large plasmid, explaining the lack of significant differences in its occurrence in CC17 versus non-CC17 isolates (Sillanpaa et al., 2009). We further speculate that the plasmid(s) subsequently acquired multiple antibiotic resistance genes (Arias et al., 2009) and virulence determinants (e.g. $h y l_{E f m}$ gene cluster) via horizontal gene transfer [as also suggested by the striking differences in the size of pilA (fms21)-fms20-containing plasmids found in different clinical strains] and that these traits helped the host strain combat selective pressures in the hospital environment. Our ongoing efforts to completely sequence these large plasmids should unravel important insights into the evolution of these plasmids.

\section{Acknowledgements}

This work was supported in part by NIH grant R01 AI067861 from the Division of Microbiology and Infectious Diseases, NIAID, to B.E.M. D.S.K. was supported by a Summer Scholarship Award from the NIH grant T32 AI55449. C.A.A. was supported by NIH Pathway to Independence award 4 R00 AI072961 from the NIAID. D. P. was partially funded by a graduate scholarship from the Instituto Colombiano para el Desarrollo de la Ciencia y Tecnología, 'Francisco José de Caldas', Colciencias.

David S. Kim, ${ }^{1,2}$ Kavindra V. Singh, ${ }^{1,2}$ Sreedhar R. Nallapareddy, ${ }^{1,2}$ Xiang Qin, ${ }^{3}$ Diana Panesso,, ${ }^{1,4}$ Cesar A. Arias ${ }^{1,2,4}$ and Barbara E. Murray ${ }^{1,2,5}$

${ }^{1}$ Center for the Study of Emerging and ReEmerging Pathogens, University of Texas Medical School at Houston, Texas, USA

${ }^{2}$ Division of Infectious Diseases, Department of Internal Medicine, University of Texas Medical School at Houston, Texas, USA

${ }^{3}$ Human Genome Sequencing Center, Baylor College of Medicine, Houston, Texas, USA

${ }^{4}$ Molecular Genetics and Antimicrobial Resistance Unit, Universidad El Bosque, Bogota, Colombia

${ }^{5}$ Department of Microbiology and Molecular Genetics, University of Texas Medical School at Houston, Texas, USA

Correspondence: Barbara E. Murray (bem.asst@uth.tmc.edu)

Arias, C. A. \& Murray, B. E. (2009). Antibioticresistant bugs in the 21st century - a clinical super-challenge. N Engl J Med 360, 439-443.

Arias, C. A., Panesso, D., Singh, K. V., Rice, L. B. \& Murray, B. E. (2009). Cotransfer of antibiotic resistance genes and a $h y l_{E f m}$-containing virulence plasmid in Enterococcus faecium. Antimicrob Agents Chemother 53, 4240-4246.

Barton, B. M., Harding, G. P. \& Zuccarelli, A. J. (1995). A general method for detecting and sizing large plasmids. Anal Biochem 226, 235-240.

Hendrickx, A. P., van Wamel, W. J., Posthuma, G., Bonten, M. J. \& Willems, R. J. (2007). Five genes encoding surface-exposed LPXTG proteins are enriched in hospital-adapted Enterococcus faecium clonal complex 17 isolates. J Bacteriol 189, 83218332.

Hendrickx, A. P., Bonten, M. J., van LuitAsbroek, M., Schapendonk, C. M., Kragten, A. H. \& Willems, R. J. (2008). Expression of two distinct types of pili by a hospital-acquired Enterococcus faecium isolate. Microbiology 154, 3212-3223.

Hidron, A. I., Edwards, J. R., Patel, J., Horan, T. C., Sievert, D. M., Pollock, D. A. \& Fridkin, S. K. (2008). NHSN annual update: antimicrobialresistant pathogens associated with healthcareassociated infections: annual summary of data reported to the National Healthcare Safety Network at the Centers for Disease Control and Prevention, 2006-2007. Infect Control Hosp Epidemiol 29, 996-1011.

Nallapareddy, S. R., Singh, K. V., Sillanpaa, J., Garsin, D. A., Hook, M., Erlandsen, S. L. \& Murray, B. E. (2006). Endocarditis and biofilmassociated pili of Enterococcus faecalis. J Clin Invest 116, 2799-2807.

Nallapareddy, S. R., Singh, K. V., Okhuysen, P. C. \& Murray, B. E. (2008). A functional collagen adhesin gene, $\mathrm{acm}$, in clinical isolates of Enterococcus faecium correlates with the recent success of this emerging nosocomial pathogen. Infect Immun 76, 4110-4119.

Rice, L. B., Carias, L., Rudin, S., Vael, C., Goossens, H., Konstabel, C., Klare, I., Nallapareddy, S. R., Huang, W. \& other authors (2003). A potential virulence gene, $h y l_{E f m}$, predominates in Enterococcus faecium of clinical origin. J Infect Dis 187, 508-512.

Rice, L. B., Lakticova, V., Carias, L. L., Rudin, S., Hutton, R. \& Marshall, S. H. (2009). Transferable capacity for gastrointestinal colonization in Enterococcus faecium in a mouse model. $J$ Infect Dis 199, 342-349.

Sillanpaa, J., Nallapareddy, S. R., Prakash, V. P., Oin, X., Hook, M., Weinstock, G. M. \& Murray, B. E. (2008). Identification and phenotypic characterization of a second collagen adhesin, $\mathrm{Scm}$, and genome-based identification and analysis of 13 other predicted MSCRAMMs, including four distinct pilus loci, in Enterococcus faecium. Microbiology 154, 3199-3211.

Sillanpaa, J., Prakash, V. P., Nallapareddy, S. R. \& Murray, B. E. (2009). Distribution of genes encoding MSCRAMMs and pili in clinical and natural populations of Enterococcus faecium. J Clin Microbiol 47, 896-901.

Singh, K. V., Nallapareddy, S. R. \& Murray, B. E. (2007). Importance of the ebp (endocarditisand biofilm-associated pilus) locus in the pathogenesis of Enterococcus faecalis ascending urinary tract infection. J Infect Dis 195, 16711677.

Top, J., Willems, R. \& Bonten, M. (2008).

Emergence of CC17 Enterococcus faecium: from commensal to hospital-adapted pathogen. FEMS Immunol Med Microbiol 52, 297-308.

Willems, R. J., Homan, W., Top, J., van SantenVerheuvel, M., Tribe, D., Manzioros, X., Gaillard, C., Vandenbroucke-Grauls, C. M., Mascini, E. M. \& other authors (2001). Variant esp gene as a marker of a distinct genetic lineage of vancomycin-resistant Enterococcus faecium spreading in hospitals. Lancet 357, 853-855. 